\title{
ON FUNCTIONS HOLOMORPHIC IN A STRIP REGION, AND AN EXTENSION OF WATSON'S PROBLEM
}

\author{
BY \\ S. MANDELBROJT AND G. R. MacLANE
}

1. Introduction. The following theorem is well known.

Theorem A. Let $\phi(\zeta)$ be holomorphic and bounded in $R(\zeta)>0$, and continuous on the boundary. If $\phi(\zeta)$ is not identically zero, then $\left.{ }^{1}\right)$

$$
\int^{\infty} \frac{\log |\phi(i \eta)|}{\eta^{2}} d \eta>-\infty, \quad \int_{-\infty} \frac{\log |\phi(i \eta)|}{\eta^{2}} d \eta>-\infty .
$$

In the usual statement of this theorem, for example Ostrowski [9, pp. 195$196]\left({ }^{2}\right)$, it is supposed that $\phi(\zeta)$ is holomorphic on $R(\zeta)=0$. If we note, however, that if $f(x) \not \equiv 0$ is holomorphic and bounded in $|x|<1$, and continuous on $|x|=1$ except perhaps at $x=1$, then the set of points on $|x|=1$ where $f(x)=0$ is of measure zero $\left(^{3}\right)$, it is evident that a slight modification of Ostrowski's proof yields Theorem A( $\left.{ }^{4}\right)$.

Since $\phi(\zeta)$ is bounded, (1) is equivalent to stating that the left-hand member of (1) is finite.

Conversely there follows from well known results:

THEOREM B. Let $L(u), u>0$, be an increasing function such that

$$
\int^{\infty} \frac{L(u)}{u^{2}} d u<\infty
$$

There exists a function $\phi(\zeta)$ not identically zero, holomorphic in $R(\zeta)>0$, and continuous on the boundary, such that $\log M(\rho)<-L(\rho)$ where $M(\rho)$ $=\max _{|\theta| \leqq \pi / 2}\left|\phi\left(\rho e^{i \theta}\right)\right|$.

The proof of this theorem follows immediately from Lemma VI of this

Presented to the Society, August 23, 1946; received by the editors June 10, 1946.

(1) By $\int^{\infty}<\infty\left(\int^{\infty}>-\infty\right)$ we mean that there exists a positive number $a$ such that $\lim _{N \rightarrow \infty} f_{a}^{N}=A$ exists and $A<\infty(A>-\infty)$. By $\int^{\infty}=\infty$ we mean that $A=\infty$. Similarly for $\int_{-\infty}<\infty\left(\int_{-\infty}>-\infty\right)$.

(2) Numbers in brackets refer to the references cited at the end of the paper.

(3) This (which is trivial to prove) is of course a particular case of a theorem of $F$. and M. Riesz giving the same conclusion without imposing continuity on $|x|=1$.

(4) Actually, as was proved by Szegö [10], Theorem A is still true if the condition that $\phi(\zeta)$ be continuous on the boundary is dropped. Also it should be remarked that obviously the condition $|\phi(\zeta)|<M$ may be replaced by the condition that $\phi(\zeta)$ be the quotient of two bounded functions. Szegö uses the condition (in $|x|<1$ ) that $\int_{0}^{2 \pi}\left|f\left(r e^{i \theta}\right)\right|^{2} d \theta$ be bounded for $r<1$, but it may be shown that any such function is the quotient of two bounded functions. 
paper, if we set $\zeta=e^{s}, \phi(\zeta)=F(s), L(u)=N(\log u)$.

It is convenient to translate the facts above by the transformation $\zeta=e^{x}$, whence:

THEOREM $\mathrm{A}^{\prime}$. Let $F(z)$ be holomorphic and bounded in the strip $|y|<\pi / 2$, continuous on the boundary, and not identically zero. Then the integrals

$$
\int^{\infty} \log |F(x \pm i \pi / 2)| e^{-x} d x
$$

are finite.

THEOREM B'. If $N(x), x>-\infty$, is an increasing function such that $\int^{\infty} N(x) e^{-x} d x<\infty$, then there exists a function $F(z)$, not identically zero, holomorphic in the strip $|y|<\pi / 2$, continuous on the boundary, and such that

$$
\log |F(x \pm i \pi / 2)| \leqq \log M(x) \leqq-N(x)
$$

where

$$
M(x)=\max _{|y| \leqq \pi / 2}|F(x+i y)| .
$$

The following problem was suggested by Watson: $\left\{M_{n}\right\}$ being a sequence of positive numbers, to give necessary and sufficient conditions on $\left\{M_{n}\right\}$ such that if $\phi(\zeta)$ is a function holomorphic in the half-plane $R(\zeta) \geqq 0$ and satisfies there the inequalities

$$
|\phi(\zeta)| \leqq M_{n}|\zeta|^{-n} \quad(n \geqq 1)
$$

then $\phi(\zeta)$ is identically zero.

This problem is closely related to the problem of unicity of asymptotic representation in a half-plane; that is, the solution of Watson's problem furnishes the answer to the following question: Let $\sum a_{n} \zeta^{-n}$ represent asymptotically (à la Poincaré) in the half-plane $R(\zeta) \geqq 0$ the holomorphic function $\phi(\zeta)$, this representation satisfying the inequalities

$$
\left|\phi(\zeta)-\sum_{n=0}^{m-1} \frac{a_{n}}{\zeta^{n}}\right||\zeta|^{m}<M_{m} \quad(m \geqq 1) .
$$

What are the conditions on $\left\{M_{n}\right\}$ in order that $\phi(\zeta)$ be the only function satisfying (3)?

Denjoy [3] gave a sufficient condition that (2) imply $\phi(\zeta) \equiv 0$; Carleman [2] gave a necessary and sufficient condition, and Ostrowski [9] gave an alternative proof. Actually, Ostrowski proved that if Carleman's conditions are not satisfied, then there exists a function $\phi(\zeta)$, holomorphic and not taking the value zero in $R(\zeta) \geqq 0$, and satisfying (2).

Let us suppose that $\lim M_{n}^{1 / n}=\infty$, and let us consider in the plane XOY the points $P_{n}$ with coordinates $\left(n, \log M_{n}\right)(n \geqq 1)$. Let $y=\Pi(x)$ be the enve- 
lope of all straight lines of non-negative slope passing through at least one point $P_{n}$ but lying above no points $P_{n}$. This envelope is a modified (since we consider only lines of non-negative slope) polygon of Newton; in other words, $y=\Pi(x)$ is the highest increasing convex curve under which no $P_{n}$ is situate. If we set $\log M_{n}^{c}=\Pi(n)$, Carleman's condition is equivalent to the following:

THEOREM C. If $\lim$ inf $M_{n}^{1 / n}<\infty$ and if $\phi(\zeta)$, holomorphic in $R(\zeta) \geqq 0$, satisfies (2) then $\phi(\zeta) \equiv 0$. If $\lim M_{n}^{1 / n}=\infty$, a necessary and sufficient condition that from (2) together with $\phi(\zeta)$ holomorphic in $R(\zeta) \geqq 0$ it follows that $\phi(\zeta) \equiv 0$ is that

$$
\sum \frac{M_{n}^{c}}{M_{n+1}^{c}}=\infty .
$$

This form of the condition was given by Mandelbrojt $[4,5]$; it is more suitable to the purposes of the present paper than the others. It is readily seen that the same condition is also necessary and sufficient that from (2) together with $\phi(\zeta)$ holomorphic in the half-plane $R(\zeta)>0$, continuous in $R(\zeta) \geqq 0$, it follows that $\phi(\zeta) \equiv 0$.

If we set $\zeta=e^{z}$ we have the following two theorems.

THEOREM $\mathrm{C}^{\prime}$. Let $\left\{M_{n}\right\}, n \geqq 1$, be a sequence of positive numbers. Let $F(z)$ be holomorphic in the strip $|y|<\pi / 2$, continuous on the boundary, and such that in this strip

$$
|F(z)|<M_{n} e^{-n x} \quad(n \geqq 1) .
$$

If $\lim \inf M_{n}^{1 / n}<\infty$, or if $\lim M_{n}^{1 / n}=\infty$ with $\sum M_{n}^{c} / M_{n+1}^{c}=\infty$, then $F(z) \equiv 0$.

THEOREM $C^{\prime \prime}$. If $\lim M_{n}^{1 / n}=\infty$ and if $\sum M_{n}^{c} / M_{n+1}^{c}<\infty$ then there exists a function $F(z)$ not identically zero, holomorphic in the strip $|y|<\pi / 2$, continuous on the boundary, and such that

$$
|F(z)|<M_{n} e^{-n x} \quad(n \geqq 1) .
$$

Mandelbrojt [6] proved that Theorem $\mathrm{A}^{\prime}$ still holds if the region $D$ : $|y|<\pi / 2$, is replaced by a domain $\Delta$ defined by $x>0,|y|<g(x), 0<g(x) \uparrow \pi / 2$, and $\int^{\infty}(\pi / 2-g(x)) d x<\infty$. Simple examples show that the conclusion is invalid when $\int^{\infty}(\pi / 2-g(x)) d x=\infty$. The condition that this integral be finite means that the area of the region $D-\Delta$ in the right half-plane be finite. Since the above results are used in many branches of the theory of functions, it seems useful to give analogous theorems valid when this area is not finite. Some restricted results of this nature were given by Mandelbrojt [7]. It is our purpose here to give general theorems (I, III) which contain Mandelbrojt's results and which essentially cannot be improved since we also prove the converse (II). These theorems lead to a solution (Theorems IV, V) of a problem generalizing that of Watson to regions other than a parallel strip. 
Since we do not suppose a finite area for $D-\Delta$, it is natural that the expressions involved in the statements of our theorems should contain a function characterizing that part of $\Delta$ contained in $R(z)<x$. Also we shall not restrict ourselves to $g(x) \leqq \pi / 2$, but will assume $g(x) \rightarrow \pi / 2$.

2. Extensions of Theorems $\mathbf{A}^{\prime}$ and $\mathbf{B}^{\prime}$. Let $g(\sigma)>0$ be defined and continuous for $\sigma \geqq a$, where $-\infty \leqq a<\infty, g(\sigma)$ of bounded variation in $(a, \infty)$, and $\lim _{\sigma \rightarrow \infty} g(\sigma)=\pi / 2$. Let $S(\sigma)$ be defined by

$$
S(\sigma)=\frac{\pi}{2} \int_{c}^{\sigma} \frac{d u}{g(u)}
$$

where $c>a$.

Let $D_{z}$ be the strip in the $z$-plane $(z=x+i y):|y|<\pi / 2$. Let $\Delta_{s}$ be the domain in the $s$-plane $(s=\sigma+i t): \sigma>a,|t|<g(\sigma)$. Let $\Gamma$ be the boundary of $\Delta_{s}$.

We prove first the following three theorems.

THEOREM I. Let $F(s)$, not identically zero, be holomorphic and bounded in $\Delta_{\text {, }}$ continuous in $\Delta_{s}+\Gamma$. Let $N(\sigma)$ be an increasing function such that for $\sigma$ large $\log |F(\sigma+i g(\sigma))| \leqq-N(\sigma)$. Then

$$
\int^{\infty} N(\sigma) e^{-S(\sigma)} d \sigma<\infty
$$

Note that from this statement it does not follow that $\int^{\infty} \log \mid F(\sigma$ $+i g(\sigma)) \mid e^{-S(\sigma)} d \sigma>-\infty$, since $-\log |F(\sigma+i g(\sigma))|$ is not necessarily increasing. Thus without further hypothesis on the boundary (cf. Theorem III) Theorem I does not contain exactly Theorem A'.

TheOREM II. Let $N(\sigma)$ be an increasing function such that $\int^{\infty} N(\sigma) e^{-S(\sigma)} d \sigma$ $<\infty$. Then there exists a function $F(s)$ holomorphic in $\Delta_{s}$, continuous and not taking the value zero in $\Delta_{s}+\Gamma$, such that

$$
\log |F(\sigma \pm i g(\sigma))| \leqq \log M(\sigma) \leqq-N(\sigma)
$$

holds in $\Delta_{s}+\Gamma$, where

$$
M(\sigma)=\max _{|t| \leqq g(\sigma) .}|F(\sigma+i t)| .
$$

THEOREM III. Let $\Delta_{s}$ be such that $g^{\prime}(\sigma)$ exists for $\sigma>k>a$ and satisfies for $\sigma>k$ the conditions

$$
\left|g^{\prime}(\sigma)\right|<A, \quad g^{\prime}(\sigma+h)-g^{\prime}(\sigma)>-A h \quad(h>0)
$$

for some positive constant $A$. Let $F(s)$, not identically zero, be holomorphic and bounded in $\Delta_{s}$, continuous in $\Delta_{s}+\Gamma$. Then

$$
\int^{\infty} \log |F(\sigma+i g(\sigma))| e^{-S(\sigma)} d \sigma>-\infty .
$$


3. Lemmas. It is convenient to prove several lemmas before proceeding with the proofs of the above theorems.

Let $z=z(s)=x(s)+i y(s)$ map $\Delta_{z}$ onto $D_{z}$ so that the interval $a<\sigma<\infty$ of the real axis in the $s$-plane corresponds to the real axis of the $z$-plane, $z(d)=1$, where $d>a$ is a fixed number, and $z^{\prime}(\sigma)>0$. Let $s(z)=\sigma(z)+i t(z)$ be the function inverse to $z(s)$.

LEMMA I. (1) $x(\sigma)=S(\sigma)+O(1)(\sigma \rightarrow \infty)$. (2) $|x(\sigma)-x(\sigma+i t)|=O(1)(\sigma \rightarrow \infty)$ uniformly for $s=\sigma+i t \in \Delta_{s}$. (3) $|\sigma(x+i y)-\sigma(x)|=O(1)(x \rightarrow \infty)$ uniformly for $z=x+i y \in D_{z}$. (4) $\left|x\left(\sigma_{1}\right)-x\left(\sigma_{2}\right)\right|=O\left(\left|\sigma_{1}-\sigma_{2}\right|\right)+O(1)\left(\sigma_{1}, \sigma_{2} \rightarrow \infty\right)$.

Proof. This lemma follows immediately from the distortion theorems of Ahlfors [1, pp. 7-16] concerning strip mappings, which for the particular map of $\Delta_{z}$ onto $D_{z}$ may be stated as follows : Let

$$
\begin{aligned}
x_{1}(\sigma) & =\min _{|t| \leq_{\theta}(\sigma)} x(\sigma+i t), & x_{2}(\sigma) & =\max _{|t| \leq_{\theta(\sigma)} .} x(\sigma+i t), \\
\sigma_{1}(x) & =\min _{|y| \leq \pi / 2} \sigma(x+i y), & \sigma_{2}(x) & =\max _{|y| \leq \pi / 2} \sigma(x+i y) .
\end{aligned}
$$

Then :

$$
\begin{aligned}
& \text { If } \int_{\sigma_{1}}^{\sigma_{2}} \frac{d \sigma}{g(\sigma)}>4, \text { then } \\
& x_{1}\left(\sigma_{2}\right)-x_{2}\left(\sigma_{1}\right) \geqq \frac{\pi}{2} \int_{\sigma_{1}}^{\sigma_{2}} \frac{d \sigma}{g(\sigma)}-4 \pi . \\
& x_{2}\left(\sigma_{2}\right)-x_{1}\left(\sigma_{1}\right) \leqq \frac{\pi}{2} \int_{\sigma_{1}}^{\sigma_{2}} \frac{d \sigma}{g(\sigma)}+C
\end{aligned}
$$

for some constant $C$.

$$
\int_{\sigma_{1}(x)}^{\sigma_{2}(x)} \frac{d \sigma}{g(\sigma)}<8
$$

The condition that $g(\sigma)$ be of bounded variation in $(a, \infty)$ (then also $g^{2}(\sigma)$ is of bounded variation) insures the validity of (II) with a constant $C$. The inequality (III) results from (I) upon setting $x_{1}\left(\sigma_{2}\right)=x_{2}\left(\sigma_{1}\right)$.

Part (1) of Lemma I follows from (I) and (II), if we set $\sigma_{1}=c, \sigma_{2}=\sigma$, the condition in (I) being satisfied for $\sigma$ sufficiently large since $g(\sigma) \rightarrow \pi / 2$. Part (2) follows from (II) with $\sigma_{1}=\sigma_{2}=\sigma$. Part (3) follows from (III) and the fact that $g(\sigma)$ is bounded. Part (4) follows from (II) and $g(\sigma) \rightarrow \pi / 2$.

LEMMA II. $\operatorname{Lim}_{\sigma \rightarrow \infty} d x(\sigma) / d \sigma=1$.

Proof. Let $D_{1}$ be that part of $D_{z}$ for which $x>x_{1}$, and $\Delta_{1}$ the corresponding 
part of $\Delta_{z}$ by the map $z=z(s)$. By (4), since $g(\sigma) \rightarrow \pi / 2, S(\sigma)=\sigma+o(\sigma)$. By Lemma I, (1), (2), $x(s)=\sigma+o(\sigma), \sigma \rightarrow \infty$, uniformly for $s \in \Delta_{8}$. Thus for sufficiently large $x_{1}, \Delta_{1}^{-} \subset \Delta_{1} \subset \Delta_{1}^{+}$, where we define

$$
\begin{array}{ll}
\Delta_{1}^{-}: & \sigma>2 x_{1}, \quad|t|<\inf _{\sigma>2 x_{1}} g(\sigma)=\overline{t_{1}} \leqq \pi / 2 ; \\
\Delta_{1}^{+}: & \sigma>x_{1} / 2, \quad|t|<\sup _{\sigma>x_{1} / 2} g(\sigma)=t_{1}^{+} \geqq \pi / 2 .
\end{array}
$$

Since $g(\sigma) \rightarrow \pi / 2$ as $\sigma \rightarrow \infty, t_{1}^{ \pm} \rightarrow \pi / 2$ as $x_{1} \rightarrow \infty$.

Let $\sigma_{0}=s\left(x_{0}\right)$, where $x_{0}>2 x_{1}$. The function $\zeta=f_{1}(z)=\left(e^{z}-e^{x_{0}}\right) /\left(e^{z}+e^{x_{0}}\right)$ maps $D_{z}$ into $|\zeta|<1, f_{1}\left(x_{0}\right)=0 . D_{\zeta}$, the image of $D_{1}$, contains that part of $|\zeta|<1$ exterior to the circle passing through $\zeta=\left(e^{x_{1}}-e^{x_{0}}\right) /\left(e^{x_{1}}+e^{x_{0}}\right)$ and orthogonal to $|\zeta|=1$. Thus $D_{\zeta}$ contains

$$
|\zeta|<1-\frac{2 e^{x_{1}}}{e^{x_{0}}+e^{x_{1}}}=R_{1} .
$$

The function

$$
w=f_{2}(s)=\frac{e^{s}-e^{\sigma_{0}}}{e^{s}+e^{\sigma_{0}}}
$$

maps $\Delta_{\overline{1}}^{-}$onto a region of the $w$-plane consisting of the part common to the two circles passing through -1 and +1 , making angles of $\pm t_{1}^{-}$with the real axis, and exterior to the circle orthogonal to $|w|=1$ passing through $w=\left(e^{2 x_{1}}-e^{\sigma_{0}}\right) /\left(e^{2 x_{1}}+e^{\sigma_{0}}\right)$. This domain contains the disc

$$
|w|<\min \left\{\frac{e^{\sigma_{0}}-e^{2 x_{1}}}{e^{\sigma_{0}}+e^{2 x_{1}}}, \frac{1-\cos \overline{t_{1}}}{\sin t_{1}^{-}}\right\}=R_{2} .
$$

Similarly, the image of $\Delta_{1}^{+}$by (6) is contained in

$$
|w|<\frac{1-\cos t_{1}^{+}}{\sin t_{1}^{+}}=R_{\mathbf{3}} \text {. }
$$

Thus $\Delta_{w}$, the map of $\Delta_{1}$ by (6), contains $|w|<R_{2}$ and is contained in $|w|<R_{8}$. The function

$$
\zeta=f_{3}(w)=f_{1}(z(s)), \quad s=f_{2}^{-1}(w)
$$

maps $\Delta_{w}$ onto $D_{5}$ with $f_{3}(0)=0$. It is readily seen that

$$
\left.\frac{d z}{d s}\right|_{s=\sigma}=\left.\frac{d \zeta}{d w}\right|_{w=0}
$$

which is a real positive quantity. 
Applying Schwarz' lemma to (9) and its inverse,

$$
\frac{R_{1}}{R_{3}}<\left.\frac{d \zeta}{d w}\right|_{w=0}<\frac{1}{R_{2}} .
$$

By (5), (7), (8), and (10) we have on letting $\sigma_{0}, x_{0} \rightarrow \infty$,

$$
\cot \frac{t_{1}^{+}}{2} \leqq \lim \inf \frac{d x}{d \sigma} \leqq \lim \sup \frac{d x}{d \sigma} \leqq \max \left\{1, \cot \frac{t_{1}}{2}\right\} \text {. }
$$

Allowing $x_{1} \rightarrow \infty, t_{1}^{ \pm} \rightarrow \pi / 2$, we have the lemma.

LEMMA III. Let $\Delta_{s}$ be such that $g^{\prime}(\sigma)$ exists for $\sigma>k>a$ and satisfies the conditions

$$
\left|g^{\prime}(\sigma)\right|<A, \quad g^{\prime}(\sigma+h)-g^{\prime}(\sigma)>-A h
$$

for some positive constant $A$. Then there exists a positive constant $\delta$ such that

$$
\frac{\bar{x}\left(\sigma_{2}\right)-\bar{x}\left(\sigma_{1}\right)}{\sigma_{2}-\sigma_{1}}>\delta \quad\left(0<\sigma_{2}-\sigma_{1}<1, \sigma_{1}>k\right)
$$

where $\bar{x}(\sigma)=x(\sigma+i g(\sigma))$.

Proof. A proof of the inequality (12) may be based on harmonic measure and Carleman's principle of domain-alteration (cf. Nevanlinna [8, p. 63]). Let $E\left(\mu, \sigma_{1}, \sigma_{2}\right), \mu \geqq 1$, be the ellipse in the $s$-plane with minor axis of length $2 b$ on the $\sigma$-axis, major axis of length $2 \mu b$, and passing through the points $\sigma_{1}+i g\left(\sigma_{1}\right)$ and $\sigma_{2}+i g\left(\sigma_{2}\right)$. If $\mu$, fixed, is chosen sufficiently large, then for all $\sigma_{1}, \sigma_{2}$ such that $k<\sigma_{1}<\sigma_{2}<\sigma_{1}+1, E\left(\mu, \sigma_{1}, \sigma_{2}\right)$ has the following two properties :

(1) There exists a constant $B$ such that $b<B$.

(2) The two symmetric $\operatorname{arcs} \alpha, \alpha^{\prime}$ of $E$ lying in the strip $\sigma_{1}<\sigma<\sigma_{2}$ are exterior to $\Delta_{s}$, and the complementary arcs are interior to $\Delta_{\mathbf{s}}$.

First, it is readily seen that

$$
\begin{aligned}
b^{2} & =\frac{g^{2}\left(\sigma_{1}\right)}{\mu^{2}}+\left[\frac{\sigma_{2}-\sigma_{1}}{2}+\frac{g^{2}\left(\sigma_{2}\right)-g^{2}\left(\sigma_{1}\right)}{2 \mu^{2}\left(\sigma_{2}-\sigma_{1}\right)}\right]^{2} \\
& =\frac{g^{2}\left(\sigma_{1}\right)}{\mu^{2}}+\left[\frac{\sigma_{2}-\sigma_{1}}{2}+\frac{g(\xi) g^{\prime}(\xi)}{\mu^{2}}\right]^{2}
\end{aligned}
$$

where $\sigma_{1}<\xi<\sigma_{2}$. Since $g(\sigma) \rightarrow \pi / 2,\left|g^{\prime}(\sigma)\right|<A, \mu \geqq 1$, and $\left|\sigma_{2}-\sigma_{1}\right|<1$, we have (1) with $B$ independent of the choice of $\mu \geqq 1$.

To prove (2), let $t=q(\sigma)$ be the equation of the upper half of $E$. Then if $s=\sigma_{3}$ is the center of $E$, we have for $\left|\sigma-\sigma_{3}\right|<b$ :

$$
q^{\prime \prime}(\sigma)=-\frac{\mu^{4} b^{2}}{q^{3}(\sigma)}<-\frac{\mu}{b}<-\frac{\mu}{B}
$$


and for $h>0$,

$$
q^{\prime}(\sigma+h)-q^{\prime}(\sigma)<-\frac{\mu h}{B}
$$

If $G(\sigma)=q(\sigma)-g(\sigma)$, then by (11) and (13), $G^{\prime}(\sigma+h)-G^{\prime}(\sigma)<(A-\mu / B) h$. Thus if we choose $\mu>A B, G^{\prime}(\sigma)$ is a decreasing function and since $G\left(\sigma_{1}\right)$ $=G\left(\sigma_{2}\right)=0, G(\sigma)>0$ for $\sigma_{1}<\sigma<\sigma_{2}$, and $G(\sigma)<0$ for $\sigma_{3}-b<\sigma<\sigma_{1}$ or $\sigma_{2}<\sigma<\sigma_{3}$ $+b$, which is $(2)$.

Let $s=\sigma_{3}$ be the center of $E$, and let $\omega\left(s ; \sigma_{1}, \sigma_{2}\right)$ be the harmonic function of $s$ regular in $\Delta_{s}$ with boundary value unity on the two symmetric arcs of $\Gamma$ in $\sigma_{1}<\sigma<\sigma_{2}$, and zero on the rest of $\Gamma$. Let $\omega_{1}\left(s ; \sigma_{1}, \sigma_{2}\right)$ be the harmonic function of $s$ regular in $E\left(\mu, \sigma_{1}, \sigma_{2}\right)$ assuming the boundary value unity on the arcs $\alpha, \alpha^{\prime}$ and vanishing on the complementary arcs of $E$. Then by Carleman's principle

$$
0 \leqq \omega_{1}\left(s ; \sigma_{1}, \sigma_{2}\right)<\omega\left(s ; \sigma_{1}, \sigma_{2}\right) \leqq 1
$$

for any $s$ in the common part of $E$ and $\Delta_{s}$.

Let $\zeta=f(s)$ map $E$ onto $|\zeta|<1$ with $f\left(\sigma_{8}\right)=0$. Then $f(s)$ is analytic, $f^{\prime}(s)$ $\neq 0$, on the boundary of $E$, and since $E$ is of fixed eccentricity and of bounded size $(b<B)$ there exists a positive constant $\gamma$ such that any arc of $E$ of length $\lambda$ corresponds to an arc of $|\zeta|=1$ of length not less than $\gamma \lambda$. Finally, since a harmonic function is preserved by a conformal map,

$$
\omega_{1}\left(\sigma_{3} ; \sigma_{1}, \sigma_{2}\right) \geqq \frac{1}{\pi} \gamma \lambda
$$

where $\lambda$ is the length of $\alpha$. But $\lambda>\sigma_{2}-\sigma_{1}$, and combining (14) and (15) we have

$$
\omega\left(\sigma_{3} ; \sigma_{1}, \sigma_{2}\right)>\frac{\gamma}{\pi}\left(\sigma_{2}-\sigma_{1}\right) .
$$

Let $x_{1}=\bar{x}\left(\sigma_{1}\right)=x\left(\sigma_{1}+i g\left(\sigma_{1}\right)\right), \quad x_{2}=\bar{x}\left(\sigma_{2}\right), x_{3}=x\left(\sigma_{3}\right)$. Then $\omega\left(s ; \sigma_{1}, \sigma_{2}\right)$ $=\omega_{2}\left(z ; x_{1}, x_{2}\right)$, where $z=z(s)$ and $\omega_{2}$ is the harmonic function of $z$ regular in $D_{z}$, zero on the boundary except for the segments in $x_{1}<x<x_{2}$ where it assumes the boundary value unity. It is readily seen that

$$
\omega_{2}\left(x_{3} ; x_{1}, x_{2}\right)=\frac{2}{\pi} \tan ^{-1}\left(\frac{e^{x_{2}-x_{1}}-1}{e^{x_{3}-x_{1}}+e^{x_{2}-x_{3}}}\right) .
$$

By Lemma I, $x_{3}=x_{1}+O(1)$ and $x_{2}-x_{1}=O(1)$ for $\sigma_{2}-\sigma_{1}<1$, and

$$
\omega_{2}\left(x_{3} ; x_{1}, x_{2}\right)<\frac{2}{\pi} \tan ^{-1} C\left(x_{2}-x_{1}\right)<\frac{2}{\pi} C\left(x_{2}-x_{1}\right)
$$


where $C$ is a positive constant independent of $\sigma_{1}, \sigma_{2}$. Combining (16) and (17) we have (12), with $\delta=\gamma / 2 C$.

Lemma IV. Let $N(\sigma)$ be a positive increasing function and let $\sigma(x+i \pi / 2)$ $=\bar{\sigma}(x)$. The convergence of one of the integrals

$$
\begin{aligned}
& \int^{\infty} N(\sigma) e^{-S(\sigma)} d \sigma, \\
& \int^{\infty} N(\bar{\sigma}(x)) e^{-x} d x \\
& \int^{\infty} N(\sigma(x)) e^{-x} d x
\end{aligned}
$$

entails the convergence of the other two.

Proof. By Lemma I, (3), there exists a positive constant $b$ such that $\sigma(x)-b<\bar{\sigma}(x)<\sigma(x)+b$, and by Lemma II there exists a positive constant $d$ such that for $x$ sufficiently large $\sigma(x)+b<\sigma(x+d)$ and $\sigma(x)-b>\sigma(x-d)$. Therefore for $x$ sufficiently large $\sigma(x-d)<\bar{\sigma}(x)<\sigma(x+d)$ and the convergence of $(\beta)$ is equivalent to the convergence of $(\gamma)$.

By Lemma I; (1), $S(\sigma(x))=x+O(1)$ and by Lemma II, $d \sigma / d x \rightarrow 1$. Thus from the relation

$$
\int_{T_{0}}^{T} N(\sigma) e^{-S(\sigma)} d \sigma=\int_{x\left(T_{0}\right)}^{x(T)} N(\sigma(x)) e^{-S(\sigma(x))} \frac{d \sigma}{d x} d x
$$

we see that there exists a positive constant $M$, independent of $T$, such that for $T_{0}>c$ sufficiently large:

$$
\frac{1}{M} \int_{x\left(T_{0}\right)}^{x(T)} N(\sigma(x)) e^{-x} d x<\int_{T_{0}}^{T} N(\sigma) e^{-S(\sigma)} d \sigma<M \int_{x\left(T_{0}\right)}^{x(T)} N(\sigma(x)) e^{-x} d x .
$$

Since all the integrands are positive it follows that the convergence of $(\alpha)$ is equivalent to the convergence of $(\gamma)$, which completes the proof.

Lemma V. Let $N_{1}(x), x \geqq 0, N_{1}(0)=0$, be an increasing function such that $\int{ }^{\infty} e^{-x} d N_{1}(x)<\infty$. The function

$$
P(z)=\int_{0}^{\infty}\left\{\log \left[1-\exp \left(-e^{-u+z}\right)\right]+u-z\right\} d N_{1}(u)
$$

is holomorphic in $D_{z}$, and satisfies there the inequalities

$$
\begin{array}{ll}
R P(z)<-\int_{0}^{x-\log 2} N_{1}(u) d u<-\int_{0}^{x-1} N_{1}(u) d u, & x \geqq 1 \\
R P(z)<0, & x<1 .
\end{array}
$$


Proof. For $z$ bounded, the bracket in (18) is $-e^{-u+z} / 2+O\left(e^{-2 u}\right)$. The singularities of the log term occur on the boundary of $D_{z}$. Thus (18) converges uniformly in any bounded closed subset of $D_{z}$, and $P(z)$ is holomorphic in $D_{z}$. Since log $\left|1-\exp \left(-e^{-u+z}\right)\right|+u-x<0$, the second part of (19) is true, and also we have for $|y|<\pi / 2, x \geqq 1$,

$$
\begin{aligned}
R P(z) & <\int_{0}^{A} \log \left|1-\exp \left(-e^{-u+z}\right)\right| d N_{1}(u)+\int_{0}^{A} u d N_{1}(u)-x \int_{0}^{A} d N_{1}(u) \\
& \leqq \int_{0}^{A} \log 2 d N_{1}(u)+A N_{1}(A)-\int_{0}^{A} N_{1}(u) d u-x N_{1}(A) \\
& =(\log 2+A-x) N_{1}(A)-\int_{0}^{A} N_{1}(u) d u .
\end{aligned}
$$

If we set $A=x-\log 2$, we obtain (19).

The proof of Lemma V is equivalent to Mandelbrojt's proof of Theorem $\mathrm{C}^{\prime \prime}$ [5, Lemma I, p. 342] if the strip is replaced by a half-plane, $N_{1}(x)$ being the distribution function of the sequence $\left\{-\log \mu_{n}\right\}$.

LEMMA VI. If $N(x),-\infty<x<\infty$, is an increasing function such that $\int^{\infty} N(x) e^{-x} d x<\infty$, there exists a function $F(z)$ holomorphic in $D_{z}$, continuous and never zero in $\bar{D}_{z}$, such that

$$
\log |F(z)|<-N(x) .
$$

Proof. We may assume that $N(x)>0$. If in Lemma $\mathrm{V}$ we set $N_{1}(x)$ $=\int_{0}^{x} N(u) d u$ we obtain a furttion $P(z)$ holomorphic in $D_{z}$, continuous in $\bar{D}_{z}$, and satisfying $R P(z)<0$ for all $x$, and for $x \geqq 3$,

$$
R P(z)<-\int_{0}^{x-1} N_{1}(u) d u<-N_{1}(x-2)<-N(x-3) .
$$

The function

$$
F(z)=e^{P(z+3)-N(0)}
$$

satisfies (20).

4. Proof of Theorems I, II, and III. The method of proof is now obvious.

Proof of Theorem I. Let us set $\Phi(z)=F(s(z))$. Then

$$
\log |\Phi(x+i \pi / 2)|=\log |F(\bar{\sigma}(x)+i g(\bar{\sigma}(x)))| \leqq-N(\bar{\sigma}(x)) .
$$

From Theorem $\mathrm{A}^{\prime}$ it follows that

$$
\int^{\infty} N(\bar{\sigma}(x)) e^{-x} d x<\infty,
$$

and from Lemma IV 


$$
\int^{\infty} N(\sigma) e^{-S(\sigma)} d \sigma<\infty .
$$

Proof of Theorem II. By Lemma IV, $(\gamma)$ converges, and therefore $\int \infty N(\sigma(x+d)) e^{-x} d x<\infty$ where $d$ is any real number. By Lemma VI there exists a function $\Phi(z)$ holomorphic in $D_{z}$, continuous and not taking the value zero in $\bar{D}_{z}$, such that in $\bar{D}_{z}$

$$
\log |\Phi(z)|<-N(\sigma(x+d)) .
$$

The function $F(s)=\Phi(z(s))$ is holomorphic in $\Delta_{s}$, continuous and not zero in $\Delta_{s}+\Gamma$, and satisfies in $\Delta_{s}$

$$
\log |F(s)|<-N(\sigma[x(\sigma+i t)+d]) .
$$

By Lemma I, (2), if $d$ is sufficiently large $x(\sigma+i t)+d>x(\sigma)$ for all $\sigma+i t \in \Delta_{s}$; $\sigma[x(\sigma+i t)+d]>\sigma(x(\sigma))=\sigma$, and $\log |F(s)|<-N(\sigma)$, which is the desired function.

REMARK. If we apply Theorem II to the domain $\Delta_{s}^{*}:|t|<g(\sigma)+e^{-\sigma}$, setting $S^{*}(\sigma)=(\pi / 2) \int_{c}^{\sigma}\left[g(u)+e^{-u}\right]^{-1} d u$, and noting that $S(\sigma)-S^{*}(\sigma)=O(1)$ so that the convergence of $\int^{\infty} N(\sigma) e^{-S *(\sigma)} d \sigma$ is equivalent to the convergence of $\int^{\infty} N(\sigma) e^{-S(\sigma)} d \sigma$, we have a stronger conclusion to Theorem II, namely that $F(s)$ is holomorphic and never zero in the closed domain $\Delta_{s}+\Gamma$ (with the exception of $s=\infty$ of course) and satisfies $\log M(\sigma) \leqq-N(\sigma)$.

Proof of Theorem III. We note first that if $F(s)=\Phi(z), \Phi(z)$ vanishes on the boundary of $D_{z}$ in a set of measure zero, and hence by Lemma III, $F(\sigma+i g(\sigma))$ as a function of $\sigma$ vanishes only on a set of measure zero, and $\log |\mathrm{F}(\sigma+i g(\sigma))|$ is continuous almost everywhere. Thus since $\bar{\sigma}(x)$ is a continuous increasing function we have

$$
\begin{aligned}
\int^{\infty} \log |F(\sigma+i g(\sigma))| e^{-S(\sigma)} & d \sigma \\
& =\int^{\infty} \log |\Phi(x+i \pi / 2)| \exp (-S(\bar{\sigma}(x))) d \bar{\sigma}(x),
\end{aligned}
$$

the second integral being a Stieltjes integral which by Lemmas I and III is greater than

$$
C \int^{\infty} \log |\Phi(x+i \pi / 2)| e^{-x} d x
$$

for some positive constant $C$. Applying Theorem $\mathrm{A}^{\prime}$ we have Theorem III.

5. A generalization of Watson's problem. We now prove the following two theorems.

TheOREM IV. Let $F(s)$ be holomorphic in $\Delta_{\mathbf{s}}$, and continuous in $\Delta_{\mathbf{s}}+\Gamma$. Let $\left\{M_{n}\right\}(n \geqq 1)$ be a sequence positive numbers such that in $\Delta_{\text {s }}$ 


$$
|F(s)| \leqq M_{n} e^{-n \sigma}
$$

If $\lim \inf M_{n}^{1 / n}<\infty$, or if $\lim M_{n}^{1 / n}=\infty$ with

$$
\sum \exp \left\{-S\left(\log \frac{M_{n+1}^{0}}{M_{n}^{c}}\right)\right\}=\infty
$$

then $F(s) \equiv 0$.

Theorem V. If $\lim M_{n}^{1 / n}=\infty$ and if

$$
\sum \exp \left\{-S\left(\log \frac{M_{n+1}^{c}}{M_{n}^{c}}\right)\right\}<\infty
$$

then there exists a function $F(s)$, holomorphic in $\Delta_{s}$, continuous and not taking the value zero in $\Delta_{\mathbf{c}}+\Gamma$, such that in $\Delta_{\mathrm{s}}$

$$
|F(s)| \leqq M_{n} e^{-n \sigma} \quad(n \geqq 1) .
$$

We prove first two lemmas.

LEMMA VII. Let $\left\{\nu_{n}\right\}$ be a positive increasing sequence with $\lim \nu_{n}=\infty$. Let $N(x)$ be its distribution function: $N(x)=0,0 \leqq x \leqq \nu_{1} ; N(x)=n, \nu_{n}<x \leqq \nu_{n+1}$. Let $N_{n}=\sum_{i=1}^{n} \nu_{i}$. The following equality holds:

$$
\int_{0}^{x} N(t) d t=\max _{n \geq 1}\left(n x-N_{n}\right)=m x-N_{m}
$$

where $m$ is such that $\nu_{m}<x \leqq \nu_{m+1}$.

Proof.

$$
\begin{aligned}
\int_{0}^{x} N(t) d t & =\sum_{k=1}^{m-1} k\left(\nu_{k+1}-\nu_{k}\right)+m\left(x-\nu_{m}\right) \\
& =m x-N_{m} .
\end{aligned}
$$

If $0<n<m$, then

$$
\begin{aligned}
n x-N_{n} & =\sum_{k=1}^{n-1} k\left(\nu_{k+1}-\nu_{k}\right)+n\left(x-\nu_{n}\right) \\
& =\sum_{k=1}^{n-1} k\left(\nu_{k+1}-\nu_{k}\right)+n \sum_{k=n}^{m-1}\left(\nu_{k+1}-\nu_{k}\right)+n\left(x-\nu_{m}\right) \\
& \leqq \sum_{k=1}^{m-1} k\left(\nu_{k+1}-\nu_{k}\right)+m\left(x-\nu_{m}\right)=m x-N_{m} .
\end{aligned}
$$

If $n>m$, then 


$$
\begin{aligned}
n x-N_{n} & =\sum_{k=1}^{m-1} k\left(\nu_{k+1}-\nu_{k}\right)+m\left(x-\nu_{m}\right)-\sum_{k=m+1}^{n} \nu_{k}+(n-m) x \\
& =m x-N_{m}-\sum_{k=m+1}^{n}\left(\nu_{k}-x\right) \leqq m x-N_{m},
\end{aligned}
$$

and the proof is complete.

Lemma VIII. Let $\left\{\nu_{n}\right\}$ and $N(x)$ be as in Lemma VII, and let $N_{1}(x)$ $=\int_{0}^{x} N(t) d t$. The two expressions

$$
\begin{gathered}
\sum^{\infty} e^{-S\left(v_{n}\right)}, \\
\int e^{-S(x)} N_{1}(x) d x
\end{gathered}
$$

converge or diverge together.

Proof. Since $S(c)=0$ we have

$$
\int_{c}^{T} e^{-S(t)} N(t) d t=e^{-S(T)} N_{1}(T)-N_{1}(c)+\frac{\pi}{2} \int_{c}^{T} N_{1}(t) e^{-S(t)} \frac{d t}{g(t)},
$$

and if $\int^{\infty} e^{-S(t)} N(t) d t$ converges, $\int{ }^{\infty} e^{-S(t)} N_{1}(t) d t$ converges also since $N_{1}(t)>0$ and $g(t) \rightarrow \pi / 2$. Conversely, if $\int^{\infty} e^{-S(t)} N_{1}(t) d t$ converges, then for some sequence of numbers $T_{i} \rightarrow \infty, e^{-S\left(T_{i}\right)} N_{1}\left(T_{i}\right) \rightarrow 0, \int_{c}^{T_{i}} e^{-S(t)} N(t) d t$ is bounded, and $\int e^{\infty} e^{-S(t)} N(t) d t$ converges. Thus the convergence of (25) is equivalent to the convergence of $\int^{\infty} e^{-S(t)} N(t) d t$. By a virtually identical argument, the convergence of $\int^{\infty} e^{-S(t)} N(t) d t$ is equivalent to the convergence of $\int^{\infty} e^{-S(t)} d N(t)$ $=\sum^{\infty} e^{-S\left(v_{\infty}\right)}$, which completes the proof of Lemma VIII.

Proof of Theorem IV. By (21) we have in $\Delta_{s}$,

$$
|F(s)| \leqq \inf _{n \geq 1} M_{n} e^{-n \sigma}=A(\sigma)
$$

and if $\lim \inf M_{n}^{1 / n}<\infty$, then $A(\sigma)=0$ for $\sigma$ sufficiently large and the conclusion is obvious. In case $\lim M_{n}^{1 / n}=\infty$ we have also

$$
A(\sigma)=\inf _{n \geq 1} M_{n}^{c} e^{-n \sigma} \text {. }
$$

Let $N(x)$ be the distribution function of the sequence $\left\{\nu_{n}\right\}$ with $\nu_{n}$ $=\log \left(M_{n}^{c} / M_{n-1}^{c}\right)\left(n \geqq 1, M_{0}=1\right)$, and $N_{1}(x)=\int_{0}^{x} N(x) d x$. Then, in the notation of Lemma VII,

$$
N_{n}=\log M_{1}^{c}+\log \frac{M_{2}^{c}}{M_{1}^{c}}+\cdots+\log \frac{M_{n}^{c}}{M_{n-1}^{c}}=\log M_{n}^{c},
$$

and by Lemma VII 


$$
\begin{aligned}
\log |F(s)| \leqq \log A(\sigma) & =-\sup _{n \geq 1}\left(n \sigma-\log M_{n}^{0}\right) \\
& =-\max \left(n \sigma-N_{n}\right)=-N_{1}(\sigma) .
\end{aligned}
$$

But by Lemma VIII, $\int^{\infty} e^{-S(x)} N_{1}(x) d x=\infty$, and therefore by Theorem I, $F(s) \equiv 0$.

Proof of Theorem V. Let $N(x)$ be the distribution function of $\left\{\nu_{n}\right\}$ with $\nu_{n}=\log \left(M_{n}^{c} / M_{n-1}^{c}\right)\left(n \geqq 1, M_{0}=1\right)$, and let $N_{1}(\sigma)=\int_{0}^{\sigma} N(x) d x$. By Lemma VIII, $\int{ }^{\infty} e^{-S(x)} N_{1}(x) d x<\infty$, and by Theorem II there exists a function $F(s)$ holomorphic in $\Delta_{s}$, continuous and never zero in $\Delta_{s}+\Gamma$, such that

$$
\log |F(s)| \leqq-N_{1}(\sigma) \text {. }
$$

But by Lemma VII, $N_{1}(\sigma)=\max _{n \geqq 1}\left(n \sigma-\log M_{n}^{e}\right)$. Therefore

$$
\log |F(s)| \leqq-\max _{n \geqq 1}\left(n \sigma-\log M_{n}^{c}\right) \leqq-n \sigma+\log M_{n}^{c},
$$

for all $n \geqq 1$, which proves Theorem $\mathrm{V}$.

When $\Delta_{s}$ is the strip $|t|<\pi / 2$, Theorems IV and $\mathrm{V}$ become Theorems $\mathrm{C}^{\prime}$ and $C^{\prime \prime}$, for then $S(\sigma)=\sigma-c$ and

$$
\sum \exp \left\{-S\left(\log \frac{M_{n+1}^{c}}{M_{n}^{c}}\right)\right\}=e^{c} \sum \frac{M_{n}^{c}}{M_{n+1}^{c}} .
$$

\section{REFERENCES}

1. L. Ahlfors, Untersuchungen zur Theorie der konformen Abbildung und der ganzen Funktionen, Acta Societatis Scientiarum Fennicae N.S. A vol. 1 (1930).

2. T. Carleman, Les fonctions quasi-analytiques, Paris, Gauthier-Villars, 1926.

3. A. Denjoy, Sur les fonctions quasi-analytiques de variable réele, C. R. Acad. Sci. Paris vol. 173 (1921) p. 1329.

4. S. Mandelbrojt, Séries de Fourier et classes quasi-analytiques, Paris, Gauthier-Villars, 1935.

5. - Some theorems connected with the theory of infinitely differentiable functions, Duke Math. J. vol. 11 (1944) pp. 341-349.

6. - Sur les fonctions holomorphes et bornées dans une partie d'un demi-plan, C. R. Acad. Sci. Paris vol. 222 (1946) p. 361.

7. - Sur les fonctions holomorphes dans une region infinie, C. R. Acad. Sci. Paris vol. 222 (1946) p. 1157.

8. R. Nevanlinna, Eindeutige analytische Funktionen, Berlin, 1936.

9. A. Ostrowski, Quasianalytische Funktionen und Bestimmtheit asymptotischer Entwicklungen, Acta Math. vol. 53 (1929) pp. 181-266. 244.

10. G. Szegö, Randwerte einer analytischen Funktion, Math. Ann. vol. 84 (1921) pp. 232-

The Rice Institute,

Houston, TeX. 